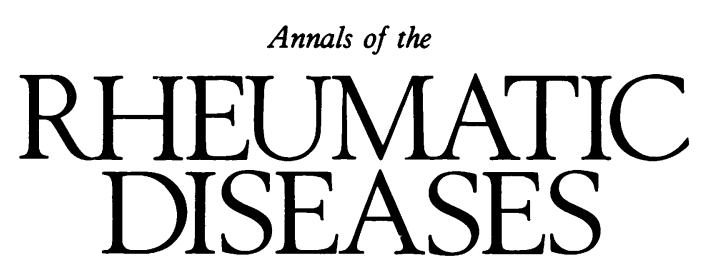

Leaders

\title{
Pregnancy and its effect on the risk of developing rheumatoid arthritis
}

Given the predominance of women over men in the occurrence of rheumatoid arthritis (RA), the well established ameliorating effect of pregnancy on the course of the disease $^{1-3}$ seems to be paradoxical. More than $75 \%$ of pregnant patients with RA improve, starting in the first or second trimester with maximum improvement in the third trimester of pregnancy. Some weeks to months after delivery, however, a disease flare up will be experienced by more than $90 \%$ of these patients. The hypothesis from these observations that female hormones may modulate the course of RA is enhanced by the protective effect of oral contraceptive use on disease development. ${ }^{4-6}$ It is thus relevant to ask whether pregnancy also influences the development of RA.

Earlier studies on the relation between parity and RA are contradictory. A large population study in the United States reported an increased rate of $R A$ among parous women above the age of 45 years compared with nulliparous women, ${ }^{7}$ whereas in a population study in the United Kingdom nulliparous women had more arthritis than the multiparous in all age groups from 35 to $65 .^{8}$ These studies, however, did not distinguish between parity preceding and following the onset of RA. Kay and Bach, ${ }^{9} 25$ years ago, were the first to make the distinction between pregnancies occurring before and after the onset of RA in a controlled study, reporting subfertility both before and after the development of RA. The subfertility seemed to be mainly due to an increased rate of nulliparity before disease onset among the patients with RA. Three recent studies looking at the effect of pregnancy history on the development of RA have confirmed these findings. ${ }^{5} 1011$ They found that women who developed RA were more often nulliparous before symptom onset than controls, indicating either that nulliparity might be a risk factor for the development of RA, or, conversely, that parity might be protective against RA. A possible 'dose-effect' relation between pregnancy experience and the risk of RA was not found: the mean family size among parous women being similar in cases and controls. An alternative interpretation for the difference is that subfertility is an early feature of RA, though preceding the onset of symptoms. In one of these studies the preventive effect of pregnancy was significantly associated with the age at first pregnancy. ${ }^{11}$ The younger the women were at their first pregnancy the more they seemed to be protected against the development of RA. The association between parity or age at first pregnancy and the risk of RA has not been confirmed in a preliminary report of a prospective study of nurses in the United States. ${ }^{12}$ In this study, however, half of the cases had been classified as probable RA, a very uncertain disease status which has therefore been abandoned in the new 1987 revised American Rheumatism Association criteria for the classification of RA. ${ }^{13}$

Despite the favourable effect of parity there is a suggestion that RA is precipitated by pregnancy as the onset seems to cluster in the postpartum period. ${ }^{14-16}$ On the other hand, several studies have shown that the risk of developing RA during pregnancy is reduced. ${ }^{614} 1617$ Del Junco found that the nearly fivefold increased risk of RA in the 12 months postpartum was counterbalanced by a decreased risk during pregnancy, ${ }^{16}$ illustrating that pregnancy only postponed the onset of RA. Some caution has to be used in interpreting data on the development of RA in the peripartum period, however, as there may be a tendency among patients to relate the onset of their symptoms to a previous critical life event. This would apply particularly when the event (child birth) requires increased physical demands and the period before that (pregnancy) had probably suppressed mild symptoms already existing.

In contrast with the possible protective effect of pregnancy on the development of RA, pregnancies with an adverse outcome have been proposed as a risk factor in the causation of RA. ${ }^{18}$ A group of mainly black American patients with RA was found to have a spontaneous abortion ratio higher than that of a comparison group before onset of their disease, ${ }^{19}$ and an increased rate of stillbirth before disease onset was found in a small number of British patients with RA compared with their unaffected relatives. ${ }^{20}$ Subsequent larger studies, however, have not confirmed these reports. $9-1121$

Summarising the epidemiological data on pregnancy and RA compiled so far we may conclude that $(a)$ pregnancy has an ameliorating effect on $\mathrm{RA}$; $(b)$ patients with $\mathrm{RA}$ are more commonly nulliparous before disease onset than are controls, but when they have children before symptom onset their family size is similar to that among controls; $(c)$ there is a suggestion that having the first pregnancy at a young age will decrease the risk of developing $R A$; (d) pregnancy possibly postpones RA onset to the postpartum period; $(e)$ it is unlikely that poor pregnancy outcome is a risk factor in rheumatoid arthritis.

Many biological mechanisms for the ameliorating effect of 
pregnancy on RA have been proposed, but a satisfactory explanation has yet to be established. The proposed mechanisms are summarised in several reviews ${ }^{1322}$ and include the immunomodulating properties of female sex hormones, immunosuppression by the various pregnancy associated proteins-for example, $\alpha_{2}$ glycoprotein, pregnancy induced suppression of the cell mediated immunity, and alterations in the glycosylation of IgG. ${ }^{23}$ These mechanisms may also explain a postponement of RA onset to the postpartum period, but the difference in parity before disease onset is not so easily explained in this way. Some gradient in risk of RA with the number of pregnancies in parous women would be expected as a 'dose-response' in such biological mechanisms. If a common immunological dysfunction is responsible for infertility and subsequent development of RA this infertility should also be reflected in a smaller family size in women who later develop RA. Protection against RA by pregnancy but independent of the number of pregnancies may otherwise suggest a threshold mechanism. A tentative explanation might be that pregnancy, particularly early in life-as a radical immunological event-incites $T$ cell repertoire changes resulting in 'vaccination' against RA. ${ }^{24}$

When interpreting data on the divergence in parity between patients with RA and controls we must also consider that pregnancy is closely related to contraception and sexual life. Oral contraception probably protects against $\mathrm{RA}$, but the lack of a dose-response relation and the many inconsistencies in the various studies have made it very difficult to find a plausible biological explanation. ${ }^{25}$ The favourable effects of the oral contraceptive pill and pregnancy were found to be mutually independent, ${ }^{511}$ indicating that women who have either been pregnant or have used the oral contraceptive pill at some time in their lives are to a certain extent protected against the development of RA. Pregnancy and oral contraceptive use have in common that they both suppress ovulation. How suppression of ovulation might lead to a longlasting immunoprotection is as yet obscure. A further common feature of pregnancy and contraception is, in general, a sexually active life. Sexual lifestyle has not yet been considered as a study objective, except perhaps for a current survey of joint disease in nuns. ${ }^{26}$ Sperm are potentially immunogenic and might incite changes in the immune system in sexually active women, particularly in the case of gastrointestinal (oral or anal) exposure to sperm. ${ }^{27}$ On the other hand, sexually transmitted diseases may play a part by evoking some kind of immunisation against RA.

These hypotheses are still speculative, however, and we await further clues to the specific part that reproduction plays in the development of RA in women.
I am most grateful to Dr Alan J Silman and Dr Peter Croft for their helpful comments on the draft manuscript.

$A R C$ Epidemiology Research Unit,

J M W HAZES

Stopford Building,

University of Manchester,

Oxford Road

Manchester M13 9PT

Correspondence to: Department of Rheumatology, University Hospital, Building 1, C2-Q, PO Box 9600, 2300 RC Leiden, The Netherlands.

1 Persellin R H. The effect of pregnancy on rheumatoid arthritis. Bull Rheum Dis 1977; 27: 922-6.

2 Cecere F. A, Persellin R H. The interaction of pregnancy and the rheumatic diseases. Clin Rheum Dis 1981; 7: 747-68.

3 Klipple G L, Cecere F A. Rheumatoid arthritis and pregnancy. Rheumatic Diseases Clinic of North America 1989; 15: 213-39.

4 Hazes J M W, Dijkmans B A C, Vandenbroucke J P, De Vries R R P, Cats A. Reduction of the risk of rheumatoid arthritis among women who take oral contraceptives. Arthritis Rheum 1990; 33: 173-9.

5 Spector T D, Roman E, Silman A J. The pill, parity and rheumatoid arthritis Arthritis Rheum 1990; 33: 782-9.

6 Koepsell T, Dugowson C, Voigt L, et al. Preliminary findings from a casecontrol study of the risk of rheumatoid arthritis in relation to oral contraceptive use. Br f Rheumatol 1989; 28 (suppl I): 41.

7 Engel A. Rheumatoid arthritis in US adults 1960-62. In: Bennett $P H$, Wood $\mathrm{P}$ H N, eds. Population studies of the rheumatic diseases. Amsterdam: Execerpta Medica, 1968: 83-9.

8 Lawrence J S. Rheumatism in populations. London: Heinemann, 1977: 245-7.

9 Kay A, Bach F. Subfertility before and after the development of rheumatoid arthritis in women. Ann Rheum Dis 1965; 24: 169-73.

10 Del Junco D J. The relationship between rheumatoid arthritis and reproductive function. Houston: University of Texas, 1988. (PhD thesis.)

11 Hazes J M W, Dijkmans B A C, Vandenbroucke J P, De Vries R R P, Cats A Pregnancy and the risk of developing rheumatoid arthritis. Arthritis Rheum (in press)

12 Hernandez-Avila M, Liang M, Willet W C, et al. Risk factors for the development of rheumatoid arthritis [Abstract]. Arthritis Rheum 1988; 31 : (suppl 4): S58.

13 Arnett F C, Edworthy S M, Bloch D A, et al. The American Rheumatism Association 1987 revised criteria for the classification of rheumatoid arthritis. Arthritis Rheum 1988; 31: 315-24.

14 Oka M. Effect of pregnancy on the onset and course of rheumatoid arthritis. Ann Rheum Dis 1953; 12: 227-9.

15 Felbo M, Svarrason E. Pregnancy and the place of therapeutic abortion in rheumatoid arthritis. Acta Obstet Gynecol Scand 1961; 40: 116-26.

16 Del Junco D J, Annegers J F, Coulam C B, Luthra H S. The relationship between rheumatoid arthritis and reproductive function. $B r \mathcal{F}$ Rheumatol 1989; 28 (suppl I): 33.

17 Short C L, Bauer W, Reynolds W E. Rheumatoid arthritis: a definition of the disease and a clinical description based on a numerical study of 293 patients and controls. Cambridge Massachusetts: Harvard University Press, 1957: 181-3.

18 Silman A J. Is pregnancy a risk factor in the causation of rheumatoid arthritis Ann Rheum Dis 1986; 45: 1031-4.

19 Kaplan D. Fetal wastage in patients with rheumatoid arthritis. $\mathcal{f}$ Rheumato 1986; 13: 875-7.

20 Silman A J, Roman E, Beral V, Brown A. Adverse reproductive outcomes in women who subsequently develop rheumatoid arthritis. Ann Rheum Dis 1988; 47: $979-81$.

21 Spector T D, Silman A J. Is poor pregnancy outcome a risk factor in rheumatoid arthritis? Ann Rheum Dis 1990; 49: 12-14.

22 Nicholas N S, Panayi G S. Rheumatoid arthritis and pregnancy. Clin Exp Rheumatol 1988; 6: 179-82.

23 Pekelharing J M, Hepp E, Kamerling J P, Gerwig G J, Leijnse B. Alterations in carbohydrate composition of serum IgG from patients with rheumatoid arthritis and from pregnant women. Ann Rheum Dis 1988; 47: 91-5.

24 Holoshitz J, Naperstek Y, Ben-Nun A, Cohen I R. Lines of T lymphocyte induce or vaccinate against autoimmune arthritis. Science 1983; 219: 56-8.

25 Hazes J M W, van Zeben D. Oral contraception and its possible protection against rheumatoid arthritis. Ann Rheum Dis 1991; 50: 72-4.

26 Mcgill P E, Brougham P A. Sex, hormones and rheumatoid arthritis: the Nun's story. Br f Rheumatol 1990; 29: 159.

27 Bronson R, Cooper C, Rosenfield D. Sperm antibodies: their role in infertility. Fertil Steril 1984; 42: 171-83.
Despite many epidemiological studies conducted in the search for environmental influences on rheumatoid arthritis (RA) we have few clues to possible risk factors. High hopes were raised following the publication of the Royal College of General Practitioners' oral contraception study, which provided evidence for a protection against RA among oral contraceptive users. ${ }^{1}$ Many subsequent studies have been initiated to question this finding. A dozen years and numerous conflicting publications later we still seem to have no clear answer to the question whether oral contraceptive use protects against RA or not. ${ }^{1-13}$ The earlier studies had methodological problems, zealously discussed in international reports. ${ }^{14-16}$ Despite methodological improvements recent studies continue to fuel the controversy. To date seven studies have shown a reduction of RA of about $50 \%$ by oral contraceptive use, ${ }^{1-7}$ whereas five studies reported no effect of oral contraceptive use on the development of $\mathrm{RA}^{8-12}$ and one was inconclusive. ${ }^{13}$ In March 1989 a special 